\title{
Holography and two phases of the QCD vacuum 8
}

\author{
B. P. Kosyakov \\ Russian Federal Nuclear Center - VNIIEF, Sarov 607190, Russia
}

\begin{abstract}
The holographic principle is often (and hastily) attributed to quantum gravity and domains of the Planck size. Meanwhile it can be usefully applied to problems where gravitation effects are negligible and domains of less exotic size. The essence of this principle is that any physical system can be taken to be either classical, placed in a D+1-dimensional spacetime, or quantum-mechanical, located in its D-dimensional boundary. For example, one believes that a hydrogen atom is a typical quantum system living in a four-dimensional spacetime, but it can also be conceived as a classical system living in a five-dimensional embracing spacetime. The subnuclear realm is more intricate since the gluon vacuum reveals two phases, the hadronic and plasma phases. They differ in energetics and symmetry. Moreover, the classical four-dimensional picture is pertinent to the behavior of constituent quarks while the plasma phase is expected to be grasped by standard four-dimensional QCD. The relation between the holographic standpoint and the symmetry treatment of these two phases is outlined. Exact retarded solutions to the classical SU(N) four-dimensional Yang-Mills equations with the source composed of several point-like colored particles is considered. Features of these solutions in the large-N limit provide insight into the gauge symmetries of two gluon vacua.
\end{abstract}

\section{Introduction}

By now, QCD is successful in the weak coupling regime, in contrast to the situation in the strong coupling regime. To restate this in a different way, one points to the happiness in high energy region and troubles with low energy region. Despite many efforts, no one managed to continue analytically between the weak and strong couplings, or between the ultraviolet and infrared energy regions. It is reasonable to anticipate a singularity preventing such a continuation. If this singularity is of the branch point type, a two-sheeted picture of the subnuclear world emerges. So, we deal with two quite different realms, cold and hot, or, C- and H-realms for short. The transition from the former to the latter is a phase transition in the thermodynamic sense [6]. It now goes under the name deconfinement. The critical energy is estimated to be $E_{c} \approx 200 \mathrm{MeV}$.

According to the general theory of phase transitions, two adjacent phases reveal different symmetries. When on the subject of a quantum-field system, the well-known theorem by Coleman [2] reads: The invariance of the system is the invariance of its ground state, the vacuum. Since vacuum averages behave classically, one should examine the symmetry of solutions to the field equations in the classical limit. In other words, the invariance of the system is the invariance of its classical background. The vacuum is filled with background fields. They form a relief relative to which quantum excitations are generated. We will see that the gauge symmetry of the H-realm is the usual QCD color-SU(3) while that of the C-realm is $S L(4, R)$, the Ne'eman-Šijački symmetry [7, 8].

These gauge symmetries should explain essential features of both phases. The central idea advocated here is as follows. A certain four-dimensional classical picture is attributed to the C-realm while the H-realm is described by a four-dimensional quantum picture, the usual QCD picture, which is in the

\footnotetext{
${ }^{1}$ Talk at the Sixth Workshop on Non-Perturbative QCD, 5-9 June 2001, American University of Paris
} 
holographic correspondence with some five-dimensional classical picture. So, geometrically, deconfinement is a topological transition augmenting the spacetime dimension by one. Furthermore, the melting of the cold phase is a quite ordinary event. It occurs whenever the acceleration of any cold quark exceeds the critical value $a_{c}$ which is of order of the critical energy $E_{c}$. We will see that deconfinement is indeed associated with a singular point on the coupling scale and a branch point on the energy scale where the spectral condition

$$
p^{2} \geq 0
$$

should have been violated but for other physical sheet, the H-realm sheet.

\section{Phenomenology}

Let us consider some well known, yet poorly understood facts. Firstly, the bulk of the hadron phenomenology is grasped by planar diagrams [10]. This implies that world lines of valence quarks are subjected to neither bifurcation nor termination (or, more precisely, the world line never takes a sharp downward turn) unless hadrons collide or decay. Such persistence of the quarks is characteristic for classical particles which are immune from creations and annihilations. The quark-antiquark sea is largely suppressed in hadrons. Thus hadrons are systems with a fixed number (two or three) of constituents. Furthermore, the Okubo-Zweig-Iizuka rule holds that creations and annihilations of quark-antiquark pairs inside hadrons are banned [9]. For example, the decay $\phi \rightarrow K+\bar{K}$ dominates over the decay into $\rho+\pi$ which goes through the $s \bar{s}$ annihilation, Fig. 11. This rule shows that cold quarks differ radically from familiar quantum-mechanical objects. Indeed, if the left diagram in Fig. 2 is allowable, then the right diagram, derivable from it by interchanging the identical quarks, with due regard for their statistics, should seemingly be also allowed but for the Okubo-Zweig-Iizuka rule. Being sufficiently rigorous, this rule provides evidence that a classical picture is pertinent to the behavior of cold quarks.

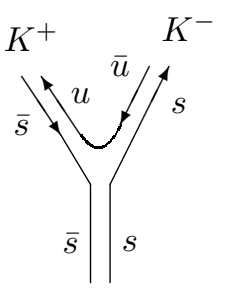

$\phi$

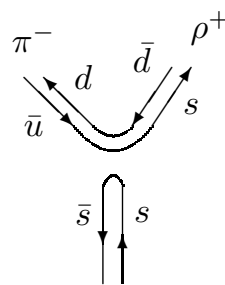

$\phi$

Figure 1: $\phi$-meson decay: Allowed (left) and forbidden (right) diagrams
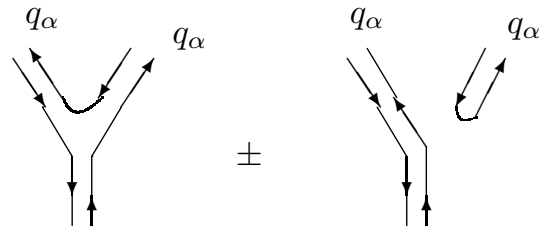

Figure 2: Decay where the identical quark exchange is taken into account

Secondly, the hadronic spectrum can be displayed in the form of the Regge trajectories on the ChewFrautschi plot of the mass squared $M^{2}$ versus the angular momentum $J$. Hadrons belonging to some Regge trajectory are separated by intervals $\Delta J=2$. Ne'eman and Šijački related this Regge arrangement to an exhaustive group classification of hadrons. Regge sequences prove to be associated with infinite multiplets of the noncompact group $S L(4, R)$. The Lie algebra $\operatorname{sl}(4, R)$ is the minimal scheme capable to explain two features of Regge trajectories: The $\Delta J=2$ rule and the infinite sequence of hadronic states. For more details see [7, 8]. To sum up, $S L(4, R)$ seems to have a direct bearing on the gauge invariance of the C-realm. 


\section{Basic principles and specific assumptions}

Since we are interesting in the gluon background fields, we should begin with the QCD equations in the limit $\hbar \rightarrow 0$. According to 't Hooft and Witten, the classical limit of QCD is related to the limit of large number of colors. One should substitute $S U(3)$ by $S U(N)$ and go to infinite $N$ to arrive at a picture where planar diagrams dominate and vacuum fluctuations of gauge invariant operators disappear. The exact form of QCD in the large $N$ limit remains in fact unknown. I suggest it to be related to the classical $S U(N)$ Yang-Mills-Wong theory as $N \rightarrow \infty$ [3].

Let us consider classical spinless point particles interacting with the classical $S U(N)$ gauge field. The particles will be called quarks and labelled by index $I$. Each quark is assigned a color charge $Q_{I}^{a}$. Let quarks be moving along timelike world lines $z_{I}^{\mu}\left(\tau_{I}\right)$ parametrized by the proper times $\tau_{I}$. This gives rise to the current

$$
j_{\mu}(x)=\sum_{I=1}^{K} \int d \tau_{I} Q_{I}\left(\tau_{I}\right) v_{\mu}^{I}\left(\tau_{I}\right) \delta^{4}\left(x-z_{I}\left(\tau_{I}\right)\right),
$$

$Q_{I}=Q_{I}^{a} T_{a}, T_{a}$ are generators of $S U(N), v_{\mu}^{I} \equiv \dot{z}_{\mu}^{I} \equiv d z_{\mu}^{I} / d \tau_{I}$ the 4 -velocity. The action is

$$
S=-\sum_{I=1}^{K} \int d \tau_{I}\left(m_{0}^{I} \sqrt{v_{\mu}^{I} v_{I}^{\mu}}+\operatorname{tr} Z_{I} \lambda_{I}^{-1} \dot{\lambda}_{I}\right)-\int d^{4} x \operatorname{tr}\left(j_{\mu} A^{\mu}+\frac{1}{16 \pi} F_{\mu \nu} F^{\mu \nu}\right) .
$$

Here, $\lambda_{I}=\lambda_{I}\left(\tau_{I}\right)$ are time-dependent elements of $S U(N), Z_{I}=e_{I}^{a} T_{a}, e_{I}^{a}$ being some constants whereby the color charge is specified, $Q_{I}=\lambda_{I} Z_{I} \lambda_{I}^{-1}$.

The Euler-Lagrange equations are the Yang-Mills equations

$$
D^{\mu} F_{\mu \nu}=4 \pi j_{\nu}
$$

the equations of motion of bare quarks

$$
m_{0}^{I} a_{I}^{\lambda}=v_{\mu}^{I} \operatorname{tr}\left(Q_{I} F^{\lambda \mu}\left(z_{I}\right)\right),
$$

where $a_{I}^{\lambda} \equiv \dot{v}_{I}^{\lambda}$ is the 4-acceleration, and the Wong equations

$$
\dot{Q}_{I}=-i g\left[Q_{I}, v_{\mu}^{I} A^{\mu}\left(z_{I}\right)\right]
$$

describing the evolution of the quark color charges. Exact solutions to this set of equations should tell us something novel about features of the gluon vacuum.

The present approach to the gluon vacuum is much different from the previous (in particular, the instanton-based one) since it takes into account the source of the classical Yang-Mills background, the classical quarks, which is of crucial importance due to the nonlinearity of the Yang-Mills dynamics.

\section{Holography}

The holographic principle in its simplest form states: There exists a correspondence between a given classical picture in a $D+1$-dimensional spacetime and a quantum picture, the $D$-dimensional hologram, that occurs in $D$-dimensional sections at any fixed instants. This finding (which went under another slogan) is due to de Alfaro, Fubini and Furlan [1. Recall their idea by the example of a system described by scalar field $\phi(x)$. Let the given quantum system be located in a $D$-dimensional Euclidean spacetime and specified by a Lagrangian $\mathcal{L}$. One introduces a fictitious time $t$. The field becomes a function of the Euclidean coordinates $x_{1}, \ldots, x_{D}$ and fictitious time $t, \phi=\phi(x, t)$. If $\frac{1}{2}(\partial \phi / \partial t)^{2}$ is treated as the kinetic term, and $\mathcal{L}$ the potential energy term, then one defines a new Lagrangian

$$
\tilde{\mathcal{L}}=\frac{1}{2}(\partial \phi / \partial t)^{2}-\mathcal{L}
$$


generating the evolution in $t$. The associated Hamiltonian is

$$
\tilde{\mathcal{H}}=\frac{1}{2} \pi^{2}+\mathcal{L}
$$

$\pi=\partial \tilde{\mathcal{L}} / \partial \dot{\phi}=\partial \phi / \partial t$ is the conjugate momentum obeying the classical Poisson bracket

$$
\{\phi(x, t), \pi(y, t)\}=\delta^{D}(x-y) .
$$

It is easy to see that the Gibbs average for an ensemble with the temperature $k T=\hbar$

$$
\mathcal{Z}[J]=\int \mathcal{D} \pi \mathcal{D} \phi \exp \left(-\frac{1}{k T} \int d^{D} x(\tilde{\mathcal{H}}+J \phi)\right)
$$

turns to the generating functional for the quantum Green functions

$$
Z[J]=\int \mathcal{D} \phi \exp \left(-\frac{1}{\hbar} \int d^{D} x(\mathcal{L}+J \phi)\right)
$$

upon taking the Gaussian integral over $\pi$. The holographic mapping of the bulk picture onto the screen picture in the spacetime sections at any instants $t$ is ensured by the Liouville theorem: Although $\phi(x, t)$ and $\pi(x, t)$ evolve in $t$, the elementary volume in phase space $\mathcal{D} \pi \mathcal{D} \phi$ and the Gibbs average $\mathcal{Z}$ are $t$ independent.

Thus, it is meaningless to ask whether a given realm is classical or quantum. It may appear both as classical and quantum, but these two looks pertain to spacetimes of nearby dimensions. To identify the realm, one should only indicate $D$. The conventional procedure of quantization only shifts the seat to another realm: In lieu of the initial classical system in $D$ dimensions, a new classical system in $D+1$ dimensions emerges.

The holography provides insight into the origin of quantum anomalies [5]. Symmetries of classical Lagrangians may be sensitive to the dimension; some of them are feasible only for a single $D^{\star}$, while another only for $D=2 n$. On the other hand, given a quantized $D^{\star}$-dimensional theory, we deal actually with the holographic image of classical $D^{\star}+1$-dimensional theory, and the symmetry under examination is missing from it.

For example, the invariance under the conformal transformations $g_{\mu \nu} \rightarrow e^{2 \varepsilon} g_{\mu \nu}$ is known to be attained if the energy-momentum tensor is traceless, $\Theta^{\mu}{ }_{\mu}=0$. In the classical $D+1$-dimensional YangMills theory,

$$
\Theta^{\mu \nu} \propto\left(F^{\mu \alpha} F_{\alpha}{ }^{\nu}+\frac{1}{4} \eta^{\mu \nu} F_{\alpha \beta} F^{\alpha \beta}\right) .
$$

The condition $\Theta^{\mu}{ }_{\mu}=0$ is fulfilled only for $D+1=4$, that is Yang-Mills equations are conformal invariant only in a $4 \mathrm{D}$ spacetime. The quantization of the classical 4D Yang-Mills theory culminates in the classical 5 D Yang-Mills theory where $\Theta_{\mu}^{\mu} \neq 0$. This is how the conformal anomaly and dimensional transmutation in QCD occurs.

\section{Solutions to the Yang-Mills-Wong equations}

There are two classes of exact retarded solutions to the Yang-Mills equations with point sources [3]. Solutions of one class are complex-valued with respect to the Cartan basis of $s u(N)$, but it is possible to convert them to the real form. Then such solutions become invariant under $S L(N, R)$ or its subgroups. Solutions of another class are real-valued and invariant under $S U(N)$.

The Cartan-Weyl basis of the Lie algebra $s u(N)$ is spanned by the set of $N^{2}$ matrices with $N$ elements of the Cartan subalgebra, $H_{n}$, satisfying the relation $\sum_{n=1}^{N} H_{n}=0$, and $N^{2}-N$ raising and lowering elements $E_{m n}^{+}$and $E_{m n}^{-}$. The nontrivial commutators are

$$
\left[H_{m}, E_{m n}^{ \pm}\right]= \pm E_{m n}^{ \pm}, \quad\left[E_{m n}^{+}, E_{m n}^{-}\right]=H_{m}-H_{n}, \quad\left[E_{k l}^{ \pm}, E_{l m}^{ \pm}\right]= \pm E_{k m}^{ \pm}
$$


One can verify that the Yang-Mills equations are satisfied by

$$
A_{\mu}=\mp \frac{2 i}{g} \sum_{I=1}^{K}\left(H_{I} \frac{v_{\mu}^{I}}{\rho_{I}}+g \kappa E_{I K+1}^{ \pm} R_{\mu}^{I} \prod_{I=1}^{K-1} \delta\left(R^{K} \cdot R^{I}\right)\right) .
$$

Here, $\kappa$ is an arbitrary real constant, a lightlike vector $R_{\mu}^{I}=x_{\mu}-z_{\mu}^{I}$ is drawn from the point of emission of the retarded signal on $I$ th worldline, $z_{\mu}^{I}$, to the point of observation, $x_{\mu}, \rho_{I}=R^{I} \cdot v^{I}$ is the retarded distance between these points, and $v_{\mu}^{I}$ is taken at the retarded instant $\tau_{I}^{\text {ret }}$. This solution describes a background field generated by some $K$-quark cluster in the cold phase. In addition, there are exact solutions describing the Yang-Mills background generated by several quark clusters.

One can define $(K+1)^{2}$ traceless imaginary matrices $\mathcal{H}_{n}$ and $\mathcal{E}_{m n}^{ \pm}$

$$
\mathcal{H}_{n} \equiv i H_{n}, \quad \mathcal{E}_{m n}^{ \pm} \equiv i E_{m n}^{ \pm}
$$

which are elements of the Lie algebra $s l(K+1, R)$. The above solutions become real valued with respect to this basis. The solutions built from $J^{2}$ such elements obeying the closed set of commutation relations are invariant under $S L(J, R), J \leq K+1$. In particular, the Yang-Mills field of a three-quark cluster is invariant under $S L(4, R)$, and that of a two-quark cluster is invariant under $S L(3, R)$. Since $S L(3, R)$ is a subgroup of $S L(4, R)$, the background of every hadron is specified by the gauge group $S L(4, R)$. This symmetry is independent of $N$ and is retained in the limit $N \rightarrow \infty$.

For $\kappa=0$, the Yang-Mills equations linearize, and we find a Coulomb-like solution corresponding the background field in the hot phase,

$$
A_{\mu}=\sum_{I=1}^{K} \sum_{n=1}^{N} e_{I}^{n} H_{n} \frac{v_{\mu}^{I}}{\rho_{I}}
$$

with arbitrary $e_{I}^{n}$. The gauge symmetry of this solution is $S U(N)$.

As is shown in [- 4 , in spacetimes of dimension different from 4, there is no exact retarded solutions other than Coulomb-like. The reason is quite simple. Only in $4 \mathrm{D}$, both differentiation and multiplication by $A_{\mu}$ raise the singularity power by one, and hence both terms of the covariant derivative $\partial_{\mu}-i g A_{\mu}$ act coherently.

\section{Discussion}

Any background generated by a cold quark occupies individually some color $s l(2, R)$ cell. Neither of two backgrounds generated by different quarks can be contained in the same $s l(2, R)$ cell. This is similar to the Pauli blocking principle. Just as a cell of volume $h^{3}$ in phase space might be occupied by at most one fermion with a definite spin projection, so any color $s l(2, R)$ cell is intended for the background of only one quark. This color blocking guarantees the totality of color cells against shrinkage. We have actually to do with the large- $N$ situation. We thus arrived at a plausible classical $4 \mathrm{D}$ description of the C-realm. It can be holographically projected onto a $3 \mathrm{D}$ quantum picture.

By contrast, the most energetically advantageous field configuration in the hot phase is such that the color charges of quarks are lined up into a fixed direction, thereby reducing $S U(N)$ to $S U(2)$. We deal with the effectively few- $N$ case. The H-realm is described by a $4 \mathrm{D}$ perturbative quantum picture which is holographically dual to a $5 \mathrm{D}$ classical picture.

The C-realm and H-realm are linked by a topological transition which passes through singular points both on the coupling scale [which is seen from the comparison of the $g$-dependences of (12) and (14)] and on the energy scale.

In the C-realm, a dressed quark possesses the 4-momentum [3]

$$
p^{\mu}=m\left(v^{\mu}+\tau_{0} a^{\mu}\right)
$$


with

$$
\tau_{0}=\frac{2}{3 m}\left|\operatorname{tr} Q^{2}\right|=\frac{8}{3 m g^{2}}\left(1-\frac{1}{N}\right) .
$$

It follows that

$$
p^{2}=m^{2}\left(1+\tau_{0}^{2} a^{2}\right) .
$$

The dressed cold quark should turn to a tachyonic state $p^{2}<0$ if its acceleration exceeds the critical value $|a|=1 / \tau_{0}$ (which is quite common with hadron collisions). Such accelerations are within the scope of validity of classical description. Indeed, let two light quarks be separated by distance $\rho$. The critical acceleration due to the Coulomb-like color force is achieved at a distance $\rho \approx\left|\operatorname{tr} Q^{2}\right| / m$ which, in view of (16), is about $g^{-2}$ times greater than the Compton wave length of the quarks. Rather than turn to the tachyonic state, the cold quark plunges into the H-realm for the period of the hadron collision. The C-realm melts and subsequently the H-realm freezes up again whenever the acceleration of any cold quark exceeds $1 / \tau_{0}$, i. e., the energy transferred to it is about its constituent mass $m$, or the deconfinement energy $E_{c} \approx 200 \mathrm{MeV}$.

\section{Conclusion and outlooks}

Let me focus your attention on two issues.

Firstly, the classicality of the C-realm must be verified experimentally in the most direct way, for example, with the aid of the Bell inequalities. The trouble with this project is twofold. Technical aspect: it is unclear how to measure kinematical characteristics, e. g., spin projections, of individual quarks contained in hadrons. As to the conceptual point of view, the Bell inequalities in the current form are independent of the spatial dimension $D$, thereby disregarding the holographic dualism between classical and quantum pictures. One should render them sensitive to the decision between classical and quantum options by just four-dimensional classical observers.

Secondly, the background fields are generated by spinning particles, the quarks. This fact seems to be very important to account for a linkage, if it exists, between the deconfinement and the chiral symmetry restoration. For holographic reasons, there exists a finite classical four-dimensional theory of spinning particles which interact with the Yang-Mills field. It displays such a C-realm where all classical processes are reversible.

The financial support of this work by ISTC, project \# 840, is acknowledged.

\section{References}

[1] de Alfaro, V., Fubini, S., \& Furlan, G., 1981, Phys. Lett. 105 B, 462-466.

[2] Coleman, S., 1966, J. Math. Phys. 7, 787.

[3] Kosyakov, B. P., 1998, Phys. Rev. D 57, 5032. E-print hep-th/9902039.

[4] Kosyakov, B. P., 1999, Theor. Math. Phys. 199, 493.

[5] Kosyakov B. P., 2000, Phys. Lett. B 492, 349. E-print hep-th/0009071.

[6] Meyer-Ortmanns, H., 1996, Rev. Mod. Phys. 68, 473.

[7] Ne'eman, Y., and Dj. Šijački, 1988, Phys. Rev. D 37, 3267.

[8] Ne'eman, Y., and Dj. Šijački, 1993, Phys. Rev. D 47, 4133. 
[9] Ogawa, S., Sawada, S., \& Nakagawa, M., 1980, Constituent Models of Elementary Particles. Chap. 4. Tokyo: Iwanami Shoten (In Japanese).

[10] Witten, E., 1979, Nuclear Physics, B 160, 57. 